\title{
The association between ultra-processed food consumption and obesity in the ZOE PREDICT 1 cohort in the United Kingdom
}

\author{
J.Y. Lee ${ }^{1}$, E.R. Leeming ${ }^{2}$, L. Francis ${ }^{3}$, T. Spector $^{2}$, S. Berry ${ }^{1}$ and R. Gibson ${ }^{1}$ \\ ${ }^{1}$ Department of Nutritional Sciences, King's College London, London, UK, \\ ${ }^{2}$ Department of Twin Research and Genetic Epidemiology, King's College London, London, UK and \\ ${ }^{3}$ Zoe Global Ltd, London, UK
}

The NOVA system has been developed to classify foods according to their nature, extent, and purpose of industrial processing into four groups: 1) unprocessed or minimally processed foods; 2) processed culinary ingredients; 3) processed foods; and 4) ultraprocessed foods ${ }^{(1)}$. The consumption of ultra-processed foods (UPFs) is increasing drastically globally and contributes to over $50 \%$ of the energy intake in several high-income countries including the United Kingdom (UK) ${ }^{(2)}$. This study aims to assess the association between UPF consumption and indicators of obesity in the ZOE PREDICT 1 cohort. It is hypothesized that UPF consumption is associated with increased body mass index (BMI) and waist circumference (WC) and the risk of overweight, obesity, and abdominal obesity.

A secondary cross-sectional analysis of dietary and anthropometric data from a total of 987 UK-based adults aged 18-65 from the ZOE PREDICT 1 trial (2018-2019) ${ }^{(3)}$ was performed. NOVA classification based on the degree of food processing was applied to foods from 10-day weighed food diaries logged by participants via a mobile application. UPF consumption was defined as the percentage of total energy intake derived from UPFs. Participant characteristics by quintile of UPF consumption were assessed using Chi-square tests for categorical variables and linear regression for continuous variables. Linear and logistic regression models were used to evaluate the associations between quintile of UPF consumption, and BMI, WC, overweight $\left(\mathrm{BMI} \geq 25 \mathrm{~kg} / \mathrm{m}^{2}\right)$, obesity $\left(B M I \geq 30 \mathrm{~kg} / \mathrm{m}^{2}\right.$ ), and abdominal obesity (defined using ethnic- and sex-specific criteria from the International Diabetes Federation).

The mean consumption of UPFs was $53.3 \pm 0.4 \%$ of total energy intake. Carbohydrate intake, sugar intake, and sodium intake were positively correlated with UPF consumption $(\mathrm{P}<0.001$ for all variables except sodium, $\mathrm{P}=0.011$ for sodium). Age, protein intake, total fat intake, and fibre intake were negatively correlated with UPF consumption ( $\mathrm{P}<0.001$ for all variables). A $10 \%$ increase in energy intake from UPF was associated with an increase of $0.8 \mathrm{~kg} / \mathrm{m}^{2}(95 \%$ CI: $0.5,1.0)$ in BMI and $1.9 \mathrm{~cm}(95 \%$ CI: $1.4,2.5)$ in WC after adjustment for age, sex, ethnicity, total energy intake, and smoking status. In the multivariate analyses adjusted for the same variables, those in the highest quintile of UPF consumption had significantly higher odds of being overweight $(\mathrm{OR}=3.30 ; 95 \%$ CI: $2.11,5.15)$, obese $(\mathrm{OR}=2.79 ; 95 \% \mathrm{CI}: 1.57,4.94)$, and abdominally obese $(\mathrm{OR}=4.39 ; 95 \% \mathrm{CI}: 2.76,6.97)$ compared to those in the lowest quintile.

This study contributes to the growing evidence that UPF consumption is associated with obesity. It supports previous findings on the role of UPF consumption in contributing to the high prevalence of obesity in the UK. Reducing UPF consumption may bring important public health benefits.

\section{References}

1. Monteiro CA, Cannon G, Levy RB et al. (2017) Public Health Nutr 22, 936-941.

2. Elizabeth L, Machado P, Zinöcker M et al. (2020) Nutrients 12, 1955.

3. Berry SE, Valdes AM, Drew DA et al. (2020) Nat Med 26, 964-973. 